\title{
Yves Bonnefoy, sous la direction de Michèle FINCK
}

\section{Fabio Scotto}

\section{CpenEdition \\ Journals}

\section{Edizione digitale}

URL: http://journals.openedition.org/studifrancesi/16738

DOI: 10.4000/studifrancesi. 16738

ISSN: 2421-5856

\section{Editore}

Rosenberg \& Sellier

\section{Edizione cartacea}

Data di pubblicazione: 1 juillet 2019

Paginazione: 194-195

ISSN: 0039-2944

Notizia bibliografica digitale

Fabio Scotto, «Yves Bonnefoy, sous la direction de Michèle FINCK», Studi Francesi [Online], 187 (LXIII | I) | 2019, online dal 01 juillet 2019, consultato il 24 janvier 2021. URL: http://journals.openedition.org/ studifrancesi/16738; DOI: https://doi.org/10.4000/studifrancesi.16738

Questo documento è stato generato automaticamente il 24 janvier 2021.

\section{(c) (†)}

Studi Francesi è distribuita con Licenza Creative Commons Attribuzione - Non commerciale - Non opere derivate 4.0 Internazionale. 


\title{
Yves Bonnefoy, sous la direction de Michèle FINCK
}

\author{
Fabio Scotto
}

\section{NOTIZIA}

Yves Bonnefoy, sous la direction de Michèle FINCK, «Europe. Revue littéraire mensuelle», 1067, mars 2018, pp. 5-246.

1 Si deve all'amorevole cura di Michèle Finck, professoressa di letterature comparate all'Université de Strasbourg Marc Bloch, questo nuovo numero monografico della prestigiosa rivista parigina «Europe», che fa seguito al precedente da me diretto (n. 890-891, juin-juillet 2003) in occasione dell'ottantesimo compleanno del poeta.

2 Miscellanea postuma, dopo la scomparsa di Yves Bonnefoy avvenuta nel 2016, questa raccolta di contributi affronta in particolare le opere più recenti dell'autore, andando così a inaugurare nuovi percorsi critici del già considerevole catalogo a lui dedicato.

Nelle sue pagine introduttive Michèle FINCK (Séparation, réparation, obstination. Sous haute tension, pp. 3-16) propone una riflessione sulla «triade heuristique» cui rimanda il suo titolo che possa porsi come paradigma d'approccio all'opera di Bonnefoy. Se la separazione, come afferma Octavio Paz, che gli fu amico, è il tratto caratterizzante dell'era e della coscienza moderne e termine ricorrente in varie raccolte, Bonnefoy non si limita ad attestarne la presenza, ma propone una meditazione sulla sua origine, che identifica nell'orgoglio, nella rinuncia, nel dubbio e nel linguaggio le articolazioni di quella che appare, a ben vedere, una chiave di lettura della poetica di un'intera generazione, da Philippe Jaccottet a Jacques Dupin. Il poeta va oltre e nella trasmutazione cerca un superamento della separazione nella riparazione attraverso la speranza dell'Irréparable baudelairiano. Tale processo trova ne L'écharpe rouge il suo pieno compimento come compassione poetica nei confronti dei genitori, ma si vale altresì delle risorse riparatrici della traduzione, del ritmo, del suono e della musica. Infine l'ostinazione, intesa come resistenza alla tentazione del nulla, trova nell'etica 
dell'interrogazione come nell'esercizio vocale dell'articolazione poetica il suo modo di esprimere la finitudine e la volontà di non cedere alla rassegnazione del dopoguerra che fu quella della generazione poetica della stagione della rivista «L'Éphémère» e di Alberto Giacometti.

Vari interventi insistono in particolare sull'opera estrema di Bonnefoy, ovvero il romanzo-saggio autobiografico-autoanalitico L'écharpe rouge (Paris, Mercure de France, 2016), che, muovendo da una lunga poesia inedita, induce il poeta a risalire alle figure che in essa appaiono, riconducibili al poeta stesso e alle figure genitoriali e al rapporto con esse, inteso come origine della propria vocazione poetica. Pierre BRUNEL ("Une royauté même pas un jour". Pour un commentaire d'Yves Bonnefoy, pp. 67-74) propone un confronto del romanzo con Le petit prince di Saint-Exupéry per il richiamo a una sorta di sovranità dell'infanzia e per l'analogia con i cromatismi del rosso che caratterizzano tanto la sciarpa bonnefoyana quanto l'abbigliamento del Petit prince, oltre a rilevare analogie con il romanzo di André Lichtenberger Le petit roi e con poesie come La Royauté di Rimbaud. Patrick NÉE (Une mémoire en rêve. Dans les sables rouges, pp. 122-136) compie un'analisi filologica delle fonti ispiratrici del romanzo, che identifica nel racconto d'avventure di Léon Lambry Dans les sables rouges (1933), già all'origine de L'arrière-pays, oltre che in altri testi di Ferdinand Ossendowski e Alexandra David-Néel, i quali evocano scenari desertici arcaici in cui si compiono ricerche archeologiche, così da mostrare l'origine intrapsichica di queste rimemorazioni intertestuali. Pierre HUGUET (Exigence poétique et exigence critique, pp. 180-190) si propone di leggere il testo "critico" che è L'écharpe rouge al fine di comprendere in che modo tale esperienza di lettura possa modificare l'approccio critico universitario e ne deduce, anche attingendo alla propria personale esperienza di rapporti intercorsi con il poeta, che ciò sia possibile solo attraverso l'implicazione soggettiva, individuale ed esperienziale del lettore-critico chiamato a partecipare al senso dello scambio e della relazione anche umani che il testo esige, fino a fare d'esso un'ontologia.

5 Odile BOMBARDE ("Jamais je n'avais passé les lourdes grilles". La forge du père, pp. 202-215) mostra la presenza del padre nell'opera dell'autore, dai primi testi fino alla sua centralità ne L'écharpe rouge. Psicanalista e assistente di Bonnefoy al Collège de France, Bombarde illustra la complessa metapsicologia che oppone all'edipica figura materna, istitutrice del linguaggio, la figura paterna, esclusa dal linguaggio e fonte di compassione filiale, fino al portato fantasmatico della scena primaria che nel romanzo esclude il figlio dalla comprensione di quanto nell'intimità i genitori si dicono in patois. Ne deduce il ruolo nel contempo «censurant» et «censuré» del padre, che si manifesta prioritariamente nell'esercizio costante della «rature» scrittoria (Raturer outre). Gérard TITUS-CARMEL (Une soirée à l'“Infinito", pp. 216-227), poeta e pittore illustratore di vari libri di Bonnefoy, nel rievocare la presentazione milanese alla Sagrestia del Bramante dell'edizione d'artista di Deux scènes et notes conjointes, si sofferma sull'onirismo dei récits en rêve e sulla complessità d'impaginazione di un volume costantemente in fieri, per via delle continue aggiunte che il poeta vi apportava, segno di un suo instancabile dinamismo mentale e poetico.

6 Tra i restanti numerosi contributi, ricordiamo almeno Philippe JACCOTTET (Noblesse d'âme, pp. 17-18), che, nel ricordare i due amici scomparsi André du Bouchet e Yves Bonnefoy, parla per essi, nel solco di Hölderlin, «non pas d'une hauteur de vue, mais d'un regard porté vers le plus haut»; Jean-Michel MAULPoIx (Une barque chargée de fruits, pp. 29-31), che nelle barche dell'opera poetica di Bonnefoy individua il desiderio e la 
speranza di un senso espresso dal teatro della parola; Pierre DHAinaut (Je dois à Yves Bonnefoy..., pp. 38-41), che gli riconosce, in tempi nei quali la nozione stessa di poesia era stata fatta provocatoriamente oggetto d'odio, di essersene fatto apologeta pur senza nascondersi le insidie del linguaggio.

Inoltre, Jérôme тнÉLот (Pour un "Bonnefoy et Rousseau", pp. 59-66) s'interroga sulla filiazione rousseauiana di Bonnefoy, che a lui pare indiscutibile, a partire dalla nozione di soggettività che gli giunge per il tramite di Rimbaud, così come sulla caduta, che vede nel passaggio dall'infanzia al concettuale l'equivalente della perdita d'innocenza del buon selvaggio corrotto dalla società e dalla proprietà dalla quale essa scaturisce, finché la "présence» di Bonnefoy finisce col rappresentare l'equivalente del «sentiment de l'existence» di Rousseau. Specialista del rapporto fra poesia e religione in Bonnefoy, Patrick WERLY ("Changer la vie": un héritage à redéfinir, pp.113-121) situa con acume l'antropologia "religiosa" di Bonnefoy nel contesto dell'evoluzione del pensiero occidentale al fine di mostrarne la portata transtorica che si condensa nel concetto di conversione, del quale Bonnefoy abbozza la critica, e nella nozione amorosa di agape cui il poeta affida il suo desiderio di cambiare la vita per distinguerlo da ogni conversione religiosa o filosofica. Si veda qui altresì una singolare riflessione sulla "fede" né religiosa né fideistica di Bonnefoy in Sophie GUERMÈs ("Le lieu de notre seule foi", pp. 105-112), che ne esamina i luoghi ora ordinari ora edenici.

8 Tra i contributi più convincenti ricordiamo infine quello di Patrick LABARTHE (L'archéologie du "désordre", pp. 137-155), che nello studiare i meccanismi di teatralizzazione insiti nell'opera del poeta a partire dalla raccolta citata nel titolo, a ben vedere la sola opera "teatrale" di Bonnefoy, esamina le dinamiche vocali e scenografiche anche fornendone delle varianti inedite che rivelano la segreta architettura di un testo il quale molto attinge a matrici inconsce la cui eco sarà poi da ritrovarsi nei lavori successivi dell'autore. Sul fronte artistico, completano l'assieme i pregevoli saggi di Alain MADELEINE-PERDRILLAT (Remarques sur Yves Bonnefoy et la peinture, pp. 156-165), di Daniel Lançon (Poésie et photographie chez Yves Bonnefoy. Au défi d'une rencontre de l'improbable, pp. 166-176), di Michela LANDI (La musique, le souvenir. "Ensemble encore", pp. 177-179), mentre con accenti più autobiografici e commossi Jean-Yves MASSON (Yves Bonnefoy, poète de l'urgence, pp. 228-235) rammenta i suoi rapporti con Bonnefoy anche inserendone la poetica del padre su un piano di «adoption» inversa del figlio poeta nei confronti del padre anonimo operaio di ferrovia.

Dominique combe (Yves Bonnefoy, Paul Ricoeur et l'éthique de la traduction, pp. 86-94) affronta la teoria del tradurre in un confronto volto a mostrare la convergenza fra il pensiero di Ricœur e quello di Bonnefoy riguardo a un'idea condivisa di etica ed ermeneutica "positiva" del tradurre. Infine, dopo un originale contributo di Michel DEGUY (Préparatifs pour un “Tombeau d'Yves Bonnefoy", pp. 236-246, nel frattempo riapparso in volume col titolo Poèmes et Tombeau pour Yves Bonnefoy (s.l., Éditions La robe noire, 2018, 96 pp.), da non dimenticare la sempre preziosa riproposizione, come testo d'autore giustamente collocato in posizione centrale, della non inedita intervista, L'Europe, le $\mathrm{xx}^{\mathrm{e}}$ siècle, la poésie. Entretien avec Yves Bonnefoy, contributo utile a ribadire l'ampiezza d'orizzonti e la capacità di pensiero di un autore giustamente ritenuto da più parti l'erede di Baudelaire nel xx secolo. 